\title{
Therapeutic Contact Lens for Scavenging Excessive Reactive Oxygen Species on the Ocular Surface
}

\author{
Seung Woo Choi ${ }^{1}$, Bong Geun $\mathrm{Cha}^{2}$, and Jaeyun $\mathrm{Kim}^{1,2,3,4 *}$
}

${ }^{1}$ Department of Health Sciences and Technology, Samsung Advanced Institute for Health Sciences \& Technology (SAIHST), Sungkyunkwan University (SKKU), Suwon 16419, Republic of Korea

${ }^{2}$ School of Chemical Engineering, Sungkyunkwan University (SKKU), Suwon 16419, Republic of Korea

${ }^{3}$ Biomedical Institute for Convergence at SKKU (BICS), Sungkyunkwan University (SKKU), Suwon 16419, Republic of Korea

${ }^{4}$ Institute of Quantum Biophysics (IQB), Sungkyunkwan University (SKKU), Suwon 16419, Republic of Korea

*Correspondence should be addressed to J. Kim (kimjaeyun@skku.edu) 


\section{Methods}

\section{The Fourier transform infrared (FTIR) spectral analysis}

The Fourier transform infrared (FTIR) spectra of the contact lens fabricated with methacrylic acid (MAA) and ceria nanoparticles (CeNPs) (MAA+, CeNP+; $50 \mathrm{mM} \mathrm{CeNP-CL)}$ and the contact lens fabricated with MAA and without CeNPs (MAA+, CeNP- ; 0 mM CeNPCL) were obtained by an FTIR spectrometer (IFS-66/S, TENSOR27, Bruker).

\section{Homogeneity of CeNP distribution in CeNP-CL.}

Flat-shaped, $50 \mathrm{mM}$ CeNP-CL with a thickness of $150 \mu \mathrm{m}$ and a diameter of $21 \mathrm{~mm}$ was fabricated. The samples were obtained by punching with a diameter of $4 \mathrm{~mm}$ from nine sections of this CeNP-CL. Each sample was digested by hydrogen peroxide and nitric acid for pretreatment overnight. The amounts of cerium ions in the samples were determined using ICPOES.

\section{Rheological measurements}

Rheological Measurements were performed using the settings from the previous report with slight modifications. ${ }^{1}$ Flat-shaped CeNP-CLs with a diameter of $8 \mathrm{~mm}$ and cerium concentrations of 0,30 , and $50 \mathrm{mM}$ in the monomer mixture were fabricated for the rheological measurements. The thickness of the samples was $450 \mu \mathrm{m}$. The rheological analysis was performed using a rheometer ARES-G2 (TA Instruments). Storage and loss moduli were measured in oscillatory mode using an $8 \mathrm{~mm}$ parallel plate, and frequency sweep test was conducted at $1 \%$ strain, $0.5-25 \mathrm{~Hz}$ and $25^{\circ} \mathrm{C}$. 


\section{Oxygen permeability of CeNP-CLs.}

The oxygen permeability of the CeNP-CLs was calculated using the method of the previous reports showing the relationship between oxygen permeability and water content..$^{2-4}$ The oxygen permeability ( $\mathrm{D}, \mathrm{D}$ : the diffusivity of the contact lens, $k$ : the oxygen solubility of the contact lens material) of conventional hydrogels for contact lenses including polyhydroxyethylmethacrylate (PHEMA) is described as the following relationship.

$$
\mathrm{D} k=1.67 e^{0.0397 \times \text { water content }}(e: \text { the natural logarithm })
$$

\section{In vitro cell-viability test in the presence of various concentrations of CeNPs only.}

The Wong-Kilbourne derivative of Chang conjunctival epithelial cells (clone 1-5c-4) (hCECs) were maintained in an RPMI 1640 medium containing 10\% heat-inactivated FBS and 1\% penicillin/streptomycin. The human meibomian gland epithelial cells (hMGECs) were maintained in a keratinocyte-serum free medium (KSFM) supplemented with $5 \mathrm{ng} / \mathrm{mL}$ epidermal growth factor (EGF), $50 \mu \mathrm{g} / \mathrm{mL}$ bovine pituitary extract (BPE), and $1 \%$ penicillin/streptomycin. Both cells were cultured in a humidified atmosphere with $5 \% \mathrm{CO}_{2}$ at $37^{\circ} \mathrm{C}$. For the 3-(4,5-dimethylthiazol-2-yl)-2,5-diphenyl-2H-tetrazolium bromide (MTT) cellgrowth assay, hCECs and hMGECs were seeded in 24 -well plates $\left(1 \times 10^{5}\right.$ cells/well $)$ and cultured overnight in culture media. Then, CeNPs with cerium concentrations of $0,25,50$, and $100 \mu \mathrm{g} /$ well were added in $1 \mathrm{~mL}$ of culture media. After cultivation for $24 \mathrm{~h}$, the CeNPs were removed from each well by washing, and the media of $1 \mathrm{~mL}$ were added. Then, $20 \mu \mathrm{L}$ of MTT reagent was added to each well, and the cells were incubated. After $3 \mathrm{~h}$ of incubation, the media were removed, and the same volume of DMSO was added to each well. The absorbance of each well was measured at $570 \mathrm{~nm}$ using the microplate reader. All the measurements were performed at least three times. 
(a)

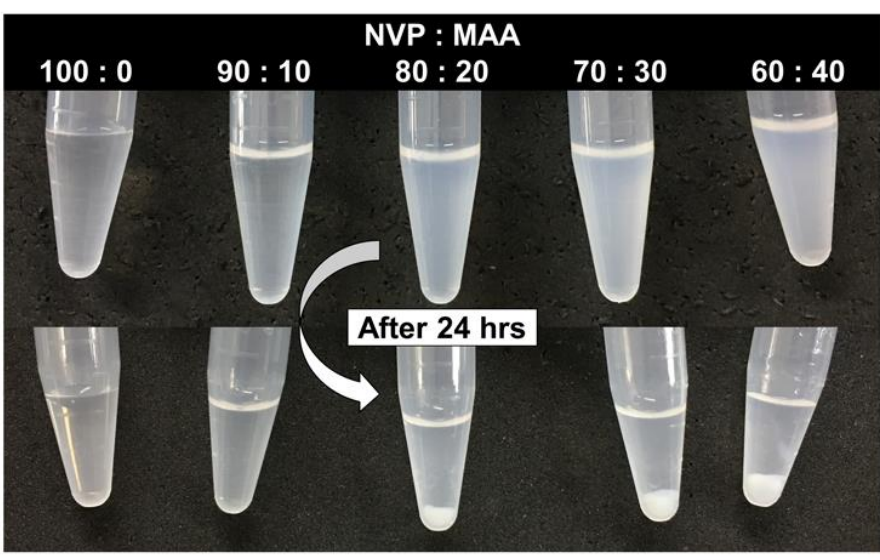

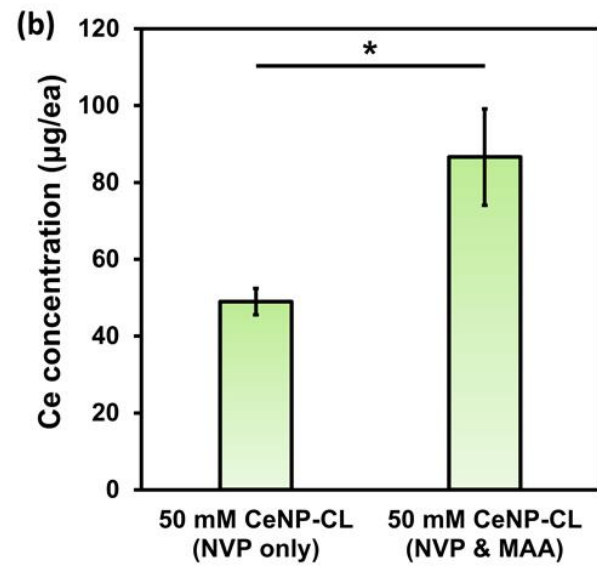

Figure S1. The effect of the addition of methacrylic acid to CeNP-CLs. (a) Aggregation according to the changes in the ratio of 1-vinyl-2-pyrrolidinone (NVP) to methacrylic acid (MAA). The solution was a homogeneous mixture of components for production of CeNP-CLs. (b) Comparison of the amounts of CeNPs in CeNP-CLs between $50 \mathrm{mM}$ CeNP-CLs with NVP only and those with NVP \& MAA (9:1). ${ }^{*} \mathrm{P}<0.05$

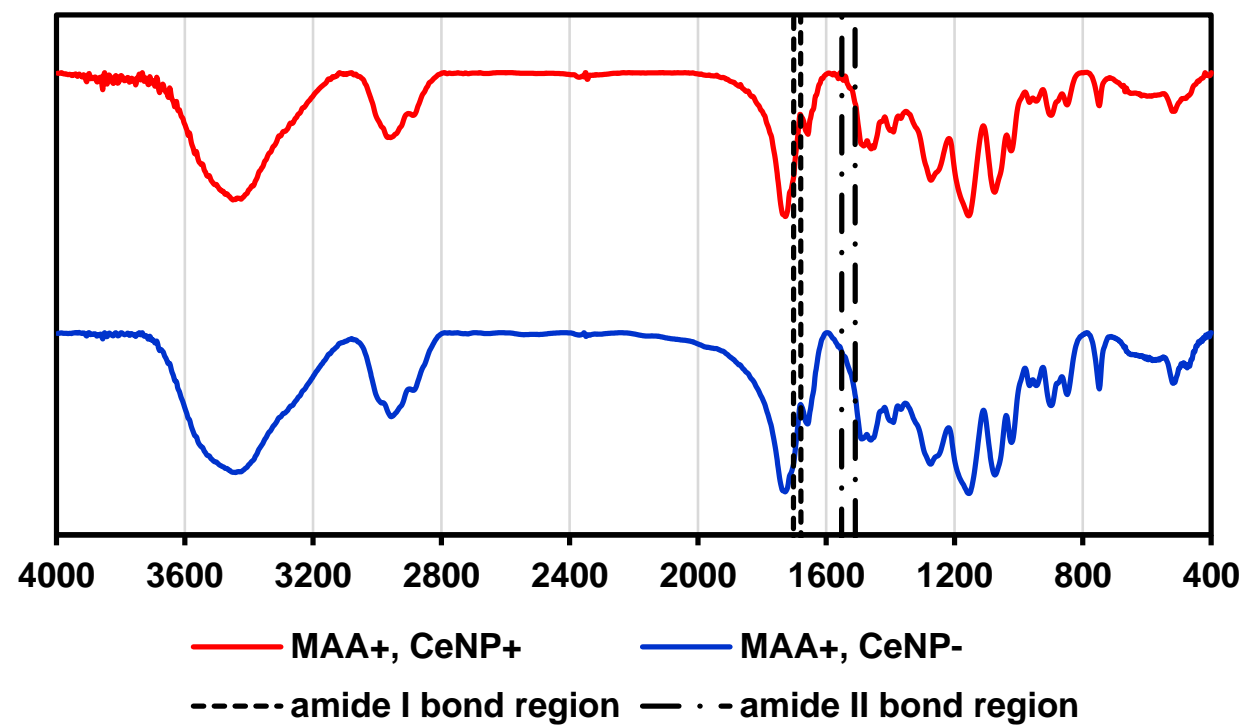

Figure S2. FTIR spectra of the contact lens containing MAA and CeNPs (red) and the contact lens containing MAA and no CeNPs (blue). 


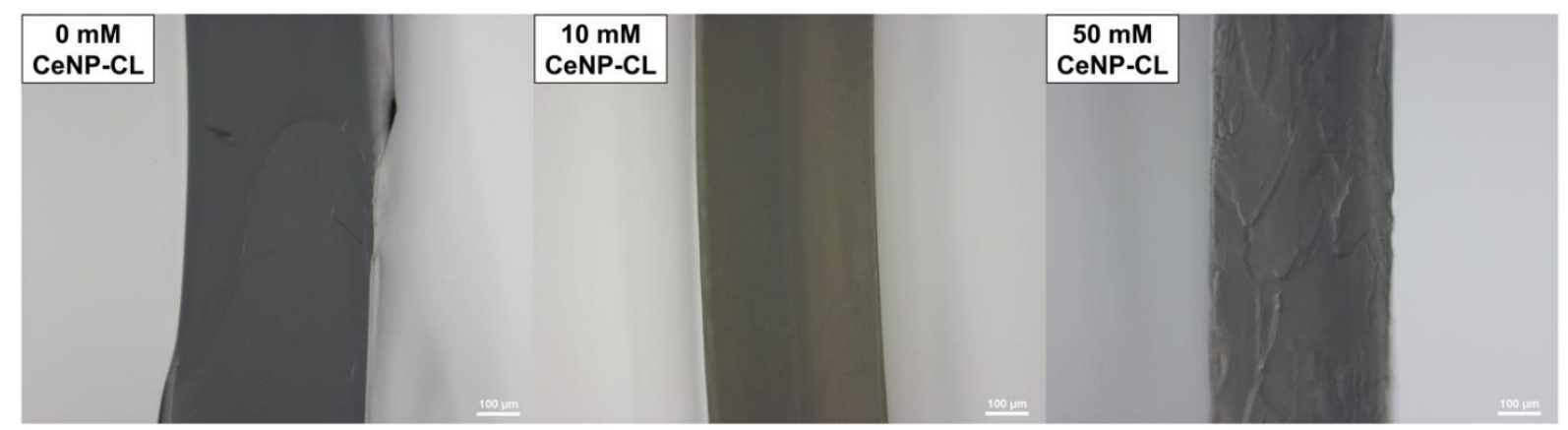

Figure S3. Photographs for the central thickness of CeNP-CLs with various CeNP concentrations $($ Scale bar $=100 \mu \mathrm{m})$.

(a)

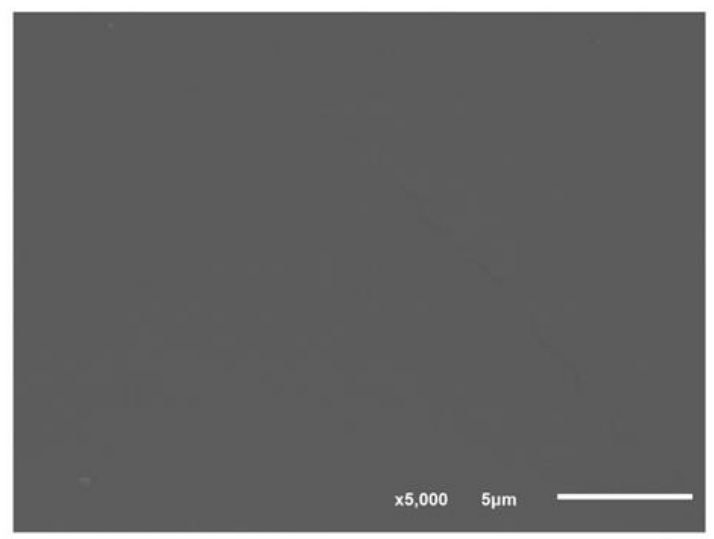

(b)

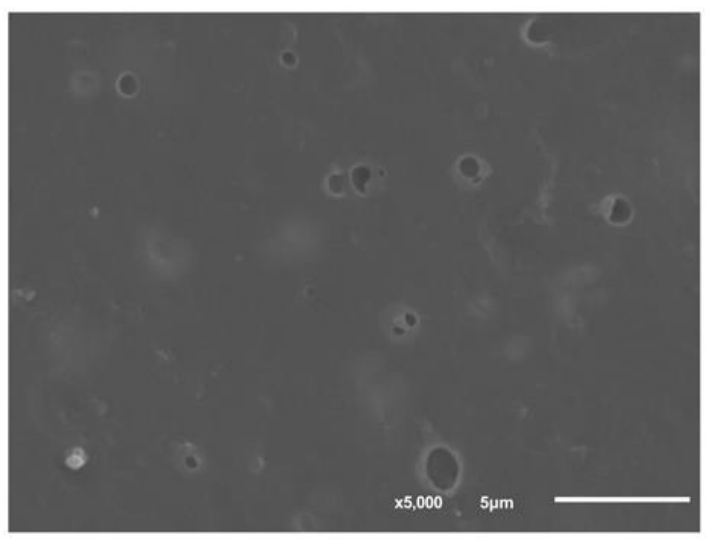

Figure S4. Scanning electron microscopic (SEM) images of (a) $50 \mathrm{mM}$ and (b) $83 \mathrm{mM} \mathrm{CeNP-}$ $\mathrm{CL}($ Scale bar $=5 \mu \mathrm{m})$.

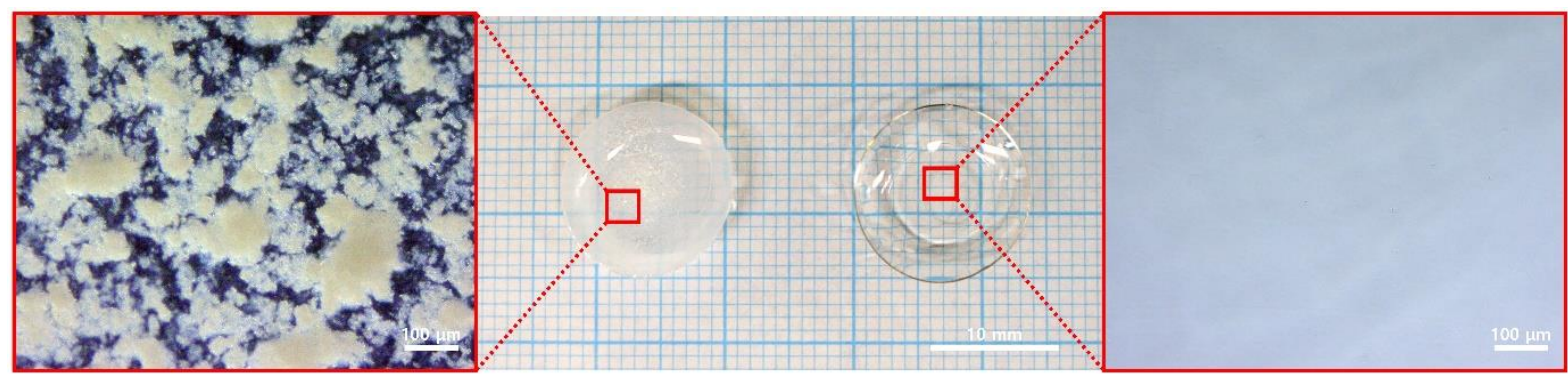

Figure S5. Gross photographs and magnified optical microscopic images of the commercially available ceria (50 mM)-embedded contact lens (left) and $50 \mathrm{mM} \mathrm{CeNP-CL} \mathrm{(right).} \mathrm{Scale} \mathrm{bars:}$ $10 \mathrm{~mm}$ for the middle and $100 \mu \mathrm{m}$ for both sides. 

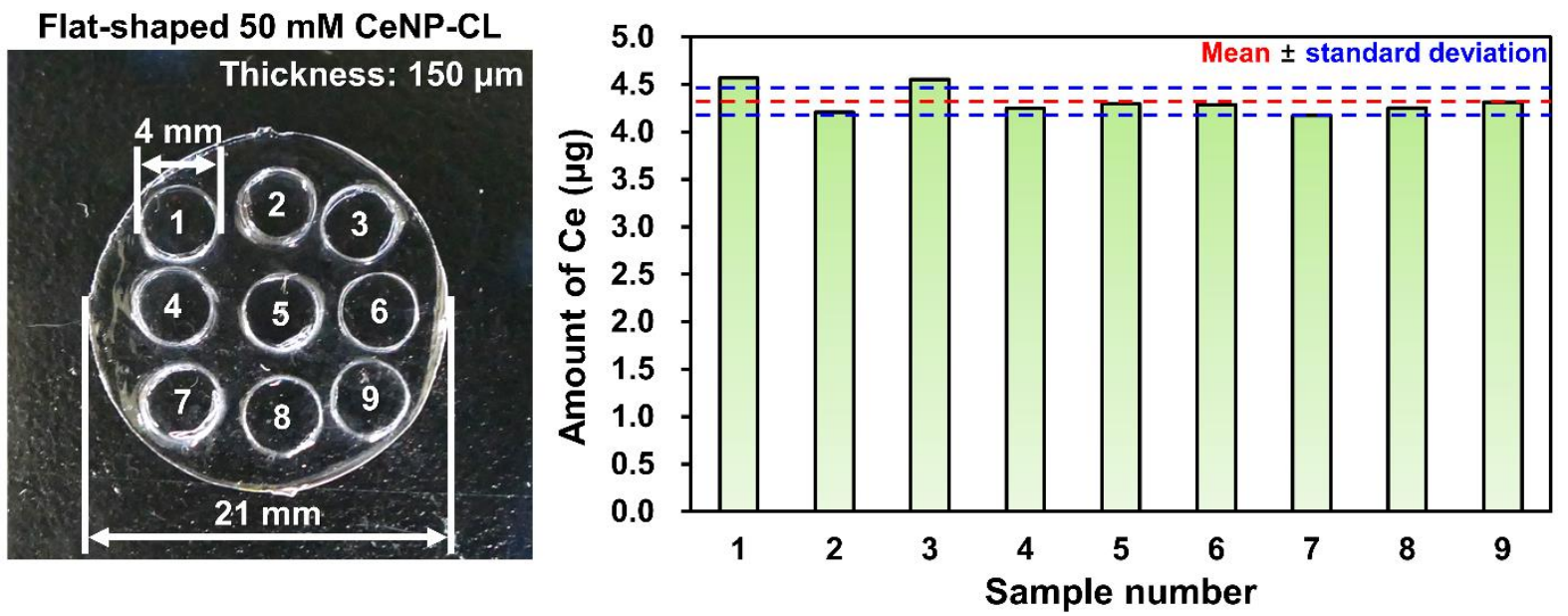

Figure S6. Comparison of the amounts of CeNPs of the samples from different sections for demonstrating homogeneity of CeNP distribution in CeNP-CL.

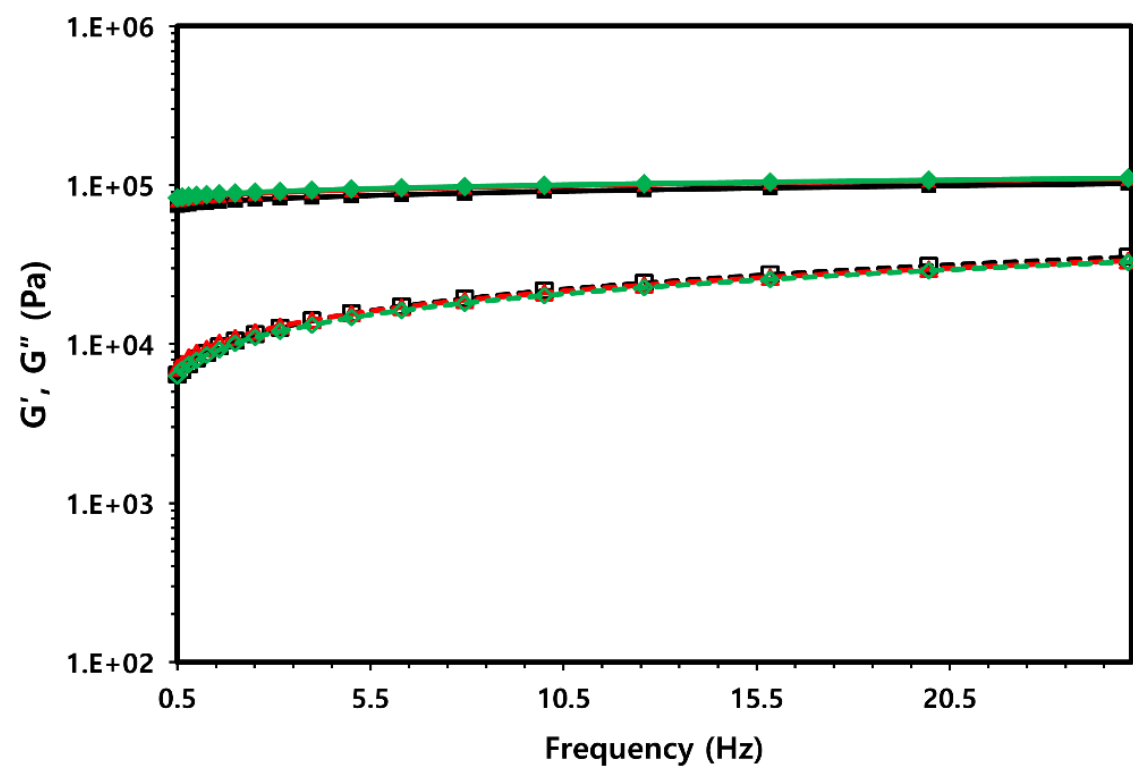

$\rightarrow \mathrm{G}^{\prime} 0 \mathrm{mM}$ CeNP-CL $\rightarrow \mathrm{G}^{\prime} 30 \mathrm{mM}$ CeNP-CL $\rightarrow \mathrm{G}^{\prime} 50 \mathrm{mM} \mathrm{CeNP}-\mathrm{CL}$

Figure S7. Frequency dependence of storage modulus $\left(\mathrm{G}^{\prime}\right)$ and loss modulus $\left(\mathrm{G}^{\prime \prime}\right)$ of CeNPCLs with various CeNP concentrations. 

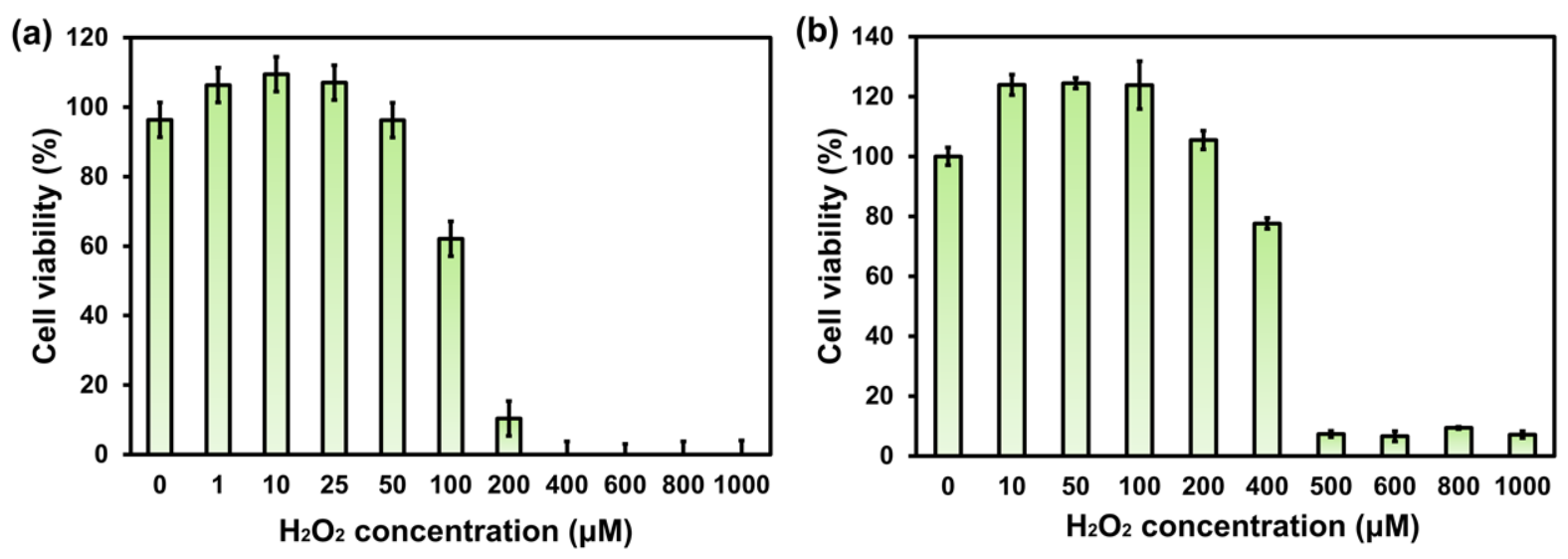

Figure S8. The viability of (a) human conjunctival epithelial cells (hCECs) and (b) human meibomian gland epithelial cells (hMGECs) in various concentrations of $\mathrm{H}_{2} \mathrm{O}_{2}$.
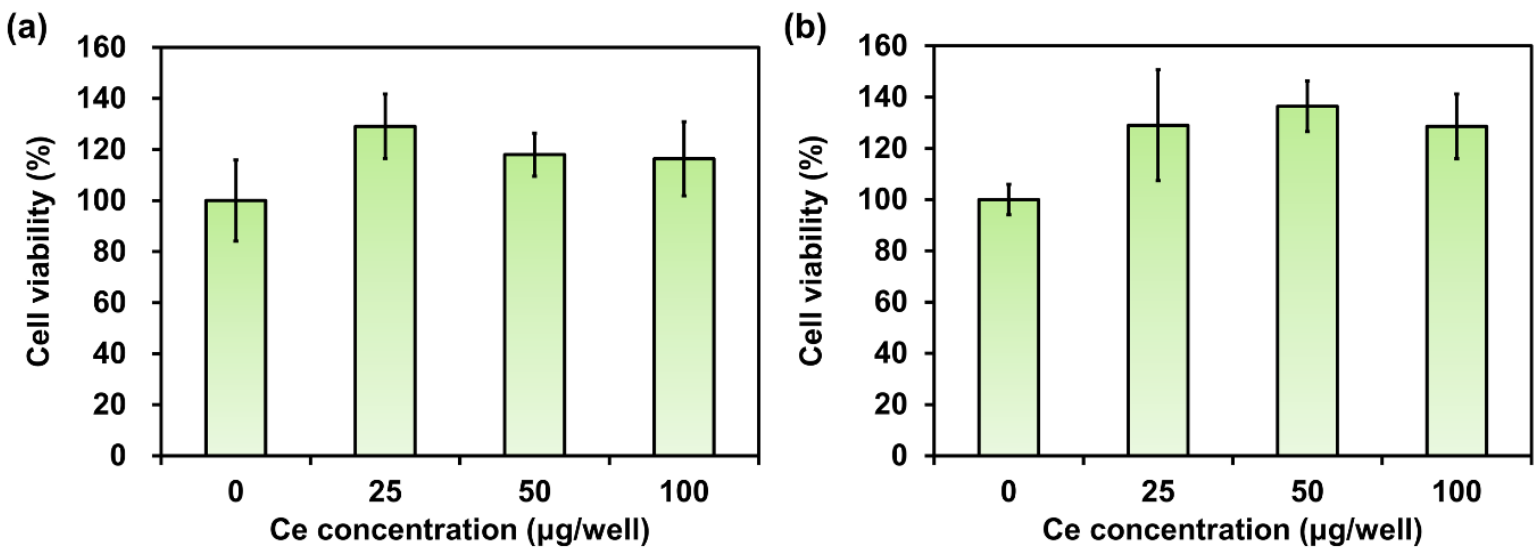

Figure S9. The viability of (a) human conjunctival epithelial cells (hCECs) and (b) human meibomian gland epithelial cells (hMGECs) in the presence of various concentrations of CeNPs only. 

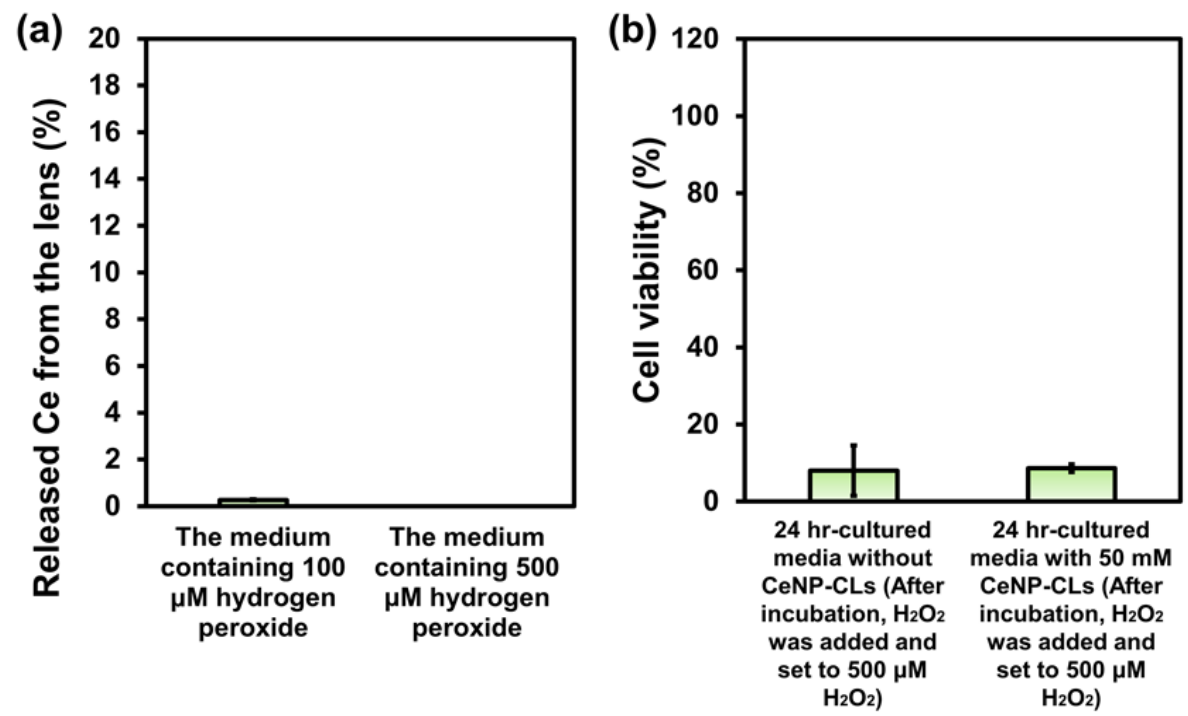

Figure S10. The data of the experiments to confirm whether CeNPs were released from CeNPCLs in various conditions. (a) Released CeNPs in the supernatants from $50 \mathrm{mM}$ CeNP-CLs, co-cultured with the $\mathrm{H}_{2} \mathrm{O}_{2}$-containing media (100 $\mu \mathrm{M}$ and $500 \mu \mathrm{M} \mathrm{H}_{2} \mathrm{O}_{2}$, respectively) without cells in $5 \% \mathrm{CO}_{2}$ at $37^{\circ} \mathrm{C}$ for 24 hours. (b) The viability of hMGECs using the supernatants of $24 \mathrm{hr}$-cultured media in $5 \% \mathrm{CO}_{2}$ at $37^{\circ} \mathrm{C}$, with or without $50 \mathrm{mM}$ CeNP-CLs. (After incubation for 24 hours and removal of CeNP-CLs, $\mathrm{H}_{2} \mathrm{O}_{2}$ was added to the media and set to $500 \mu \mathrm{M} \mathrm{H}_{2} \mathrm{O}_{2}$ ).
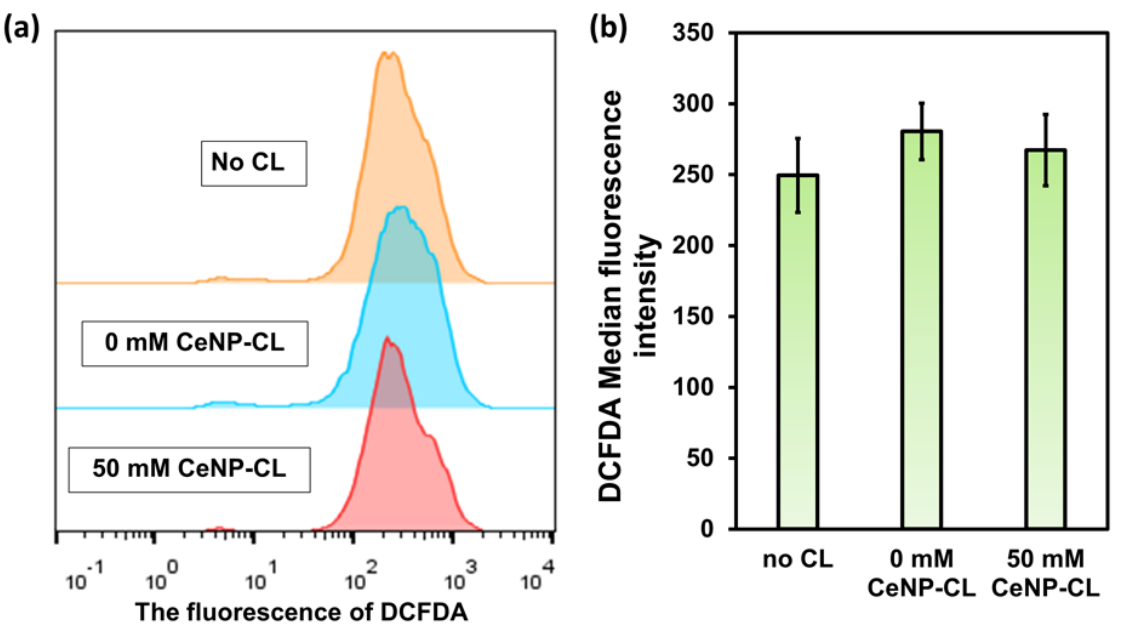

Figure S11. Evaluation of intracellular ROS scavenging properties of CeNP-CLs. (a) The results of the intracellular ROS detection assay using $2^{\prime}, 7^{\prime}$-dichlorodihydrofluorescein diacetate (DCF-DA) and CeNP-CLs, expressed by fluorescence-activated cell sorting (FACS) data. (b) The comparison of the median fluorescence intensity of DCF-DA in intracellular ROS detection assay. 
(a)

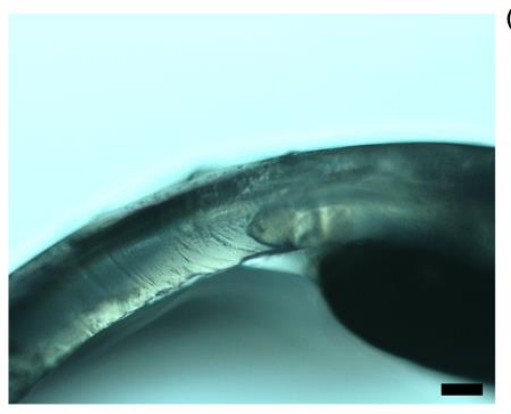

(b)

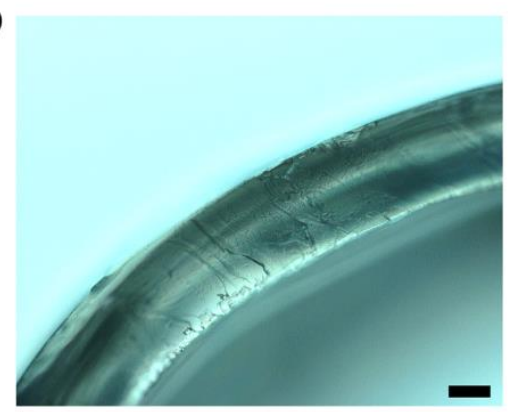

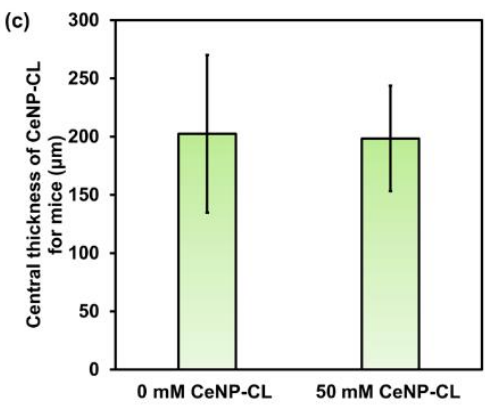

Figure S12. The central thickness data of CeNP-CLs fabricated for in vivo experiments. Photographs for the central thicknesses of (a) $0 \mathrm{mM}$ CeNP-CL and (b) $50 \mathrm{mM}$ CeNP-CL (Scale bar $=100 \mu \mathrm{m}$ ). (c) Numerical comparison data for the central thickness between $0 \mathrm{mM}$ and 50 mM CeNP-CLs.

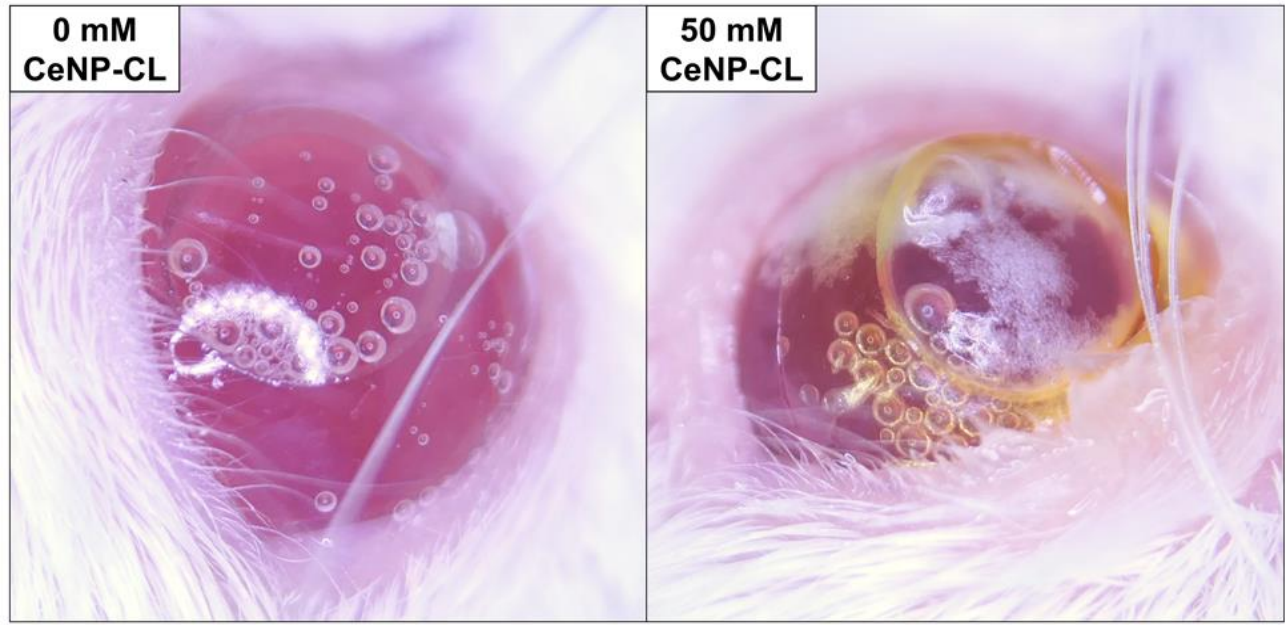

Figure S13. Photographs of immediately after instillation of $2 \mu \mathrm{L}$ of $3 \% \mathrm{H}_{2} \mathrm{O}_{2}$ solution onto the hole in CeNP-CLs on the ocular surface of mice. 

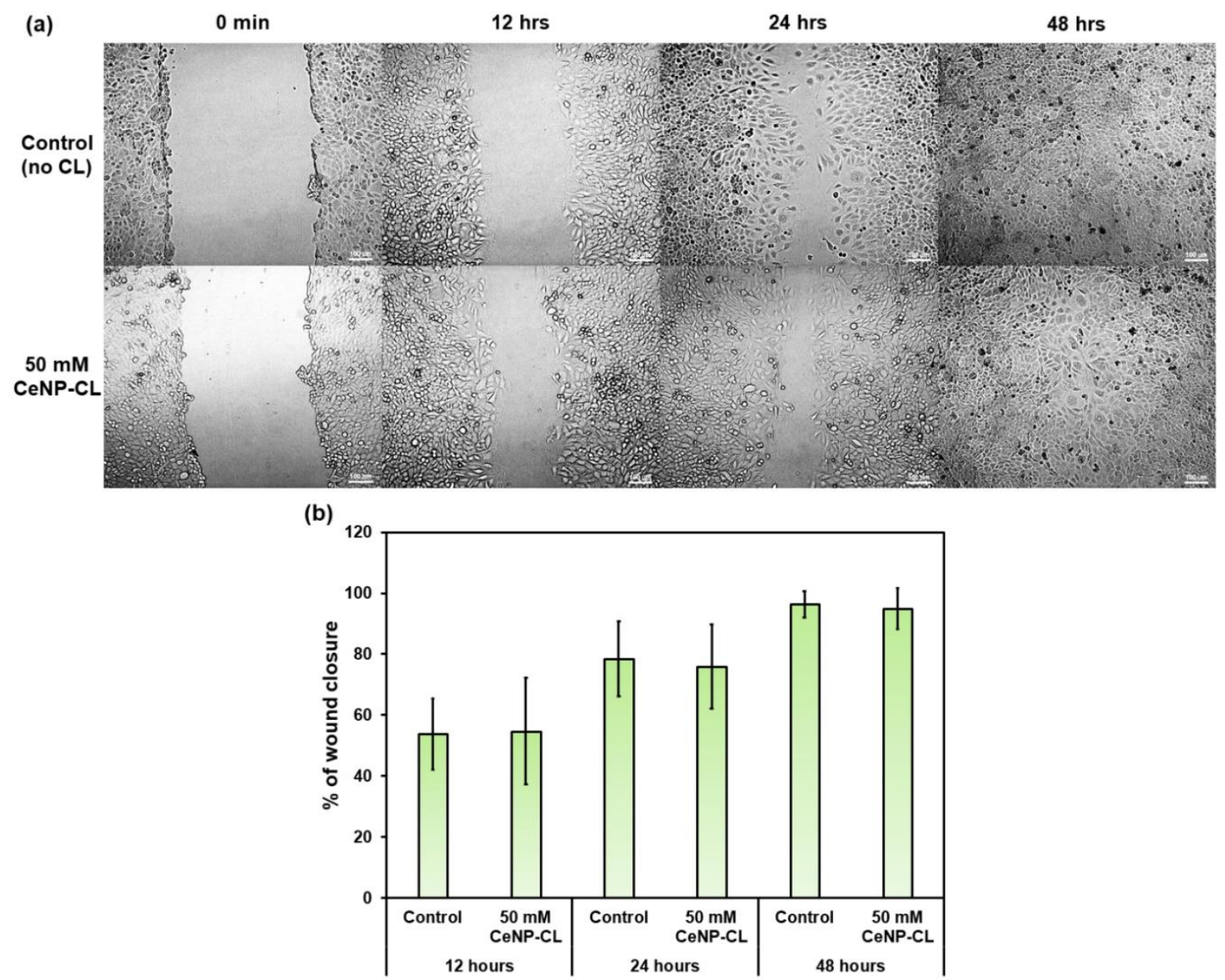

Figure S14. The result of in vitro scratch assay for wound healing. (a) Photographs during wound closure of hCECs for $48 \mathrm{hrs}$ between the groups without contact lenses and the groups with $50 \mathrm{mM}$ CeNP-CLs (Scale bar $=100 \mu \mathrm{m}$ ). (b) The numerical comparison of wound closure between the groups without contact lenses and the groups with $50 \mathrm{mM}$ CeNP-CLs.

\section{References}

1. Bhamra, T. S.; Tighe, B. J. Mechanical Properties of Contact Lenses: The Contribution of Measurement Techniques and Clinical Feedback to 50 Years of Materials Development. Cont Lens Anterior Eye 2017, 40, 70-81.

2. Morgan, P. B.; Efron, N. The Oxygen Performance of Contemporary Hydrogel Contact Lenses. Cont Lens Anterior Eye 1998, 21, 3-6.

3. Ulu, A.; Balcioglu, S.; Birhanli, E.; Sarimeseli, A.; Keskin, R.; Koytepe, S.; Ates, B. Poly(2Hydroxyethyl Methacrylate)/Boric Acid Composite Hydrogel as Soft Contact Lens Material: Thermal, Optical, Rheological, and Enhanced Antibacterial Properties. J. Appl. Polym. Sci. 2018, 135, 46575..

4. Maldonado-Codina, C. Contact Lens Practice (Third Edition), Efron, N., Ed.; Elsevier: Amsterdam, 2018; pp 45-60. 